\title{
The metabolism miracle by Diane Kress, RD CDE MNT
}

\author{
Diane Kress ${ }^{1 *}$ \\ ${ }^{1}$ Nutrition Center of Morristown, 2430 Vanderbilt Beach Road (\#108-187), Naples, FL 34109, USA
}

This is Part 1 of a 4-part series on a very relevant topic that is not being researched and is rarely taken seriously. This topic's dismissal by medical associations has caused over $60 \%$ of the US adult population to become overweight, obese, or morbidly obese. It has also triggered increased blood pressure, increased cholesterol, triglycerides, HbA1C, insulin levels, and decreased Vitamin D levels. Please read all 4 parts of this series and give yourself the opportunity to learn extremely important information that has been kept from you.

\section{A well- kept secret}

Over $60 \%$ of the adult population is in some stage of metabolic syndrome. MOST are unaware that they have this condition. A combination of genes plus environmental stressors insures that over $60 \%$ of adults eventually experience a gradual increase in body fat (especially around the middle), as well as increases in blood pressure, cholesterol, triglycerides, blood sugar, fat depositsin theliver andlow Vitamin Dlevels. These $60 \%$ also experience similar symptoms like gradually increasing fatigue, unsuccessful weight loss on low calorie diets that "used to work", inability to focus and concentrate, cravings for carbohydrate foods, belly fat, muffin top, afternoon energy slump, trouble sleeping, decreased libido, racing thoughts, etc.

\section{What is metabolic syndrome?}

Metabolic syndrome is a slow and progressive syndrome caused by an imbalance of the fat gain hormone; insulin. It is worsened by high carbohydrate intake, inadequate physical activity, stress, illness, pain, surgery, certain medications (like steroids and beta blockers), and even the normal hormonal changes that occur during puberty, pregnancy, lactation, and menopause.

\section{Metabolism $A^{\mathrm{TM}}$ vs metabolism $B^{\mathrm{Tm}}$ \\ For ease, I refer to metabolic syndrome as "METABOLISM $\mathrm{B}^{\mathrm{m} x}$." The fact is, over $60 \%$ of the population is born with the genes for metabolic syndrome. Presented with "environmental stressors" during their lifetime, they eventually develop full blown Metabolism $\mathrm{B}^{\mathrm{ma}}$. It is evidenced by their body fat, lab values, Less than $40 \%$ of the population (those who don't have the genes for metabolic syndrome) have what I refer to as Metabolism $A^{\text {тx }}$. Those with Met $\mathrm{A}^{\mathrm{m}}$ can lose weight and keep it off following a low calorie, low fat diet as they do not have insulin imbalance.}

\section{The danger is NOT knowing you have insulin imbal- ance or Met $B^{\text {ma }}$}

The following conditions and diseases are linked to insulin imbalance or Metabolism $\mathrm{B}^{\mathrm{ix}}$. The millions of people who don't know that they have Metabolism $B^{\text {tw }}$ and if they do know, don't know what to do about it, are susceptible to the following diseases: pre-diabetes, type 2 diabetes, gestational diabetes, hypoglycemia, overweight, obesity, NAFLD (fatty liver not caused by alcohol). Hypertension, hyperlipidemia, Vitamin D deficiency, breast cancer, prostate cancer, ovarian cancer, colon cancer, skin cancer, depression, anxiety disorder, GERD, sleep apnea, osteoporosis and osteopenia, PCOS, problems with fertility, Alzheimer's disease

\section{Wouldn't it be wonderful?}

Wouldn't it be incredible if the trillions of dollars of pharmaceuticals prescribed for the illnesses could be cut in half (or more) by acknowledging and promoting the correct lifestyle change (diet and exercise) that would normalize insulin release, decrease or eliminate insulin resistance, and promote hormonal equilibrium?

Wouldn't it be incredible if medical professionals and associations got out of the dark ages promoting the "calories in/calories out" mantra that is simply not valid for the majority of people with weight issues and weight related medical conditions?

Wouldn't it be incredible if those of us who understand the mechanisms involved in insulin- related disease would promote the diet and exercise program designed to match the metabolism of over $60 \%$ of the population struggling with health issues related to insulin?

Why can't medical academia and professionals admit that calories in/calories out approach does not work for $60 \%$ of people....including $60 \%$ of their peers? Is it embarrassment? Are we afraid to admit that a mistake was made back in the late 50's/early 60's when the calories in/ calories out, low fat/low cholesterol diet was crowned the gold standard?

\section{This Huge deception began over 60 years ago}

Medicine is not black/white science and research never stops. "We now know that most people with weight issues progressively produce excess fat gain hormone (insulin)". There you go, that's a simple sentence that will "cover" the errors in diet advice given over the past 60 years. A calorie based diet does NOT correct insulin imbalance. A low-fat diet is naturally higher in carbohydrate and that promotes insulin release. A low-calorie diet does help $40 \%$ of the overweight population lose weight, but $60 \%$ are getting fatter and sicker.

\section{Generations harmed by withheld Information?}

Is the fact that improper diet advice has been dispensed to generations of the population predisposing them to becoming overweight, obese, chronically ill, and tied to a myriad of medications a crime? Back in the day when metabolic syndrome was recognized, the medical associations

Correspondence to: Diane Kress RD CDE, Nutrition Center of Morristown 2430 Vanderbilt Beach Road (\#108-187), Naples, FL 34109, USA, Tel: (908)-6987893, E-mail: dietquestions@ymail.com

Received: April 28, 2017; Accepted: May 10, 2017; Published: May 13, 2017 
needed to stand up, admit the findings, and correct the teaching. It's 2017 and what you are learning is rarely spoken of. The world turns and we get fatter and sicker. The life expectancy of children born today is predicted to be shorter than the life span of their parents! Over 150 million people in the US alone have metabolic syndrome, prediabetes, or type 2 diabetes. Metabolic conditions and obesity are at epidemic levels and the epidemics have happened ever since we bought into low calorie, low fat diets for those with insulin related issues.

\section{PART 2 of Diane Kress' 4 Part Series that will CHANGE your weight, health and energy}

This is Part 2 of Diane Kress' 4 part series: Are you in the $60 \%$ ? If you were born with the predisposition to Metabolism B, you've been misled regarding weight reduction. It is vitally important to your weight, health, energy level, and wellness to determine if you do have Metabolism B and if so, learn how to eat and live in accordance with YOUR type of metabolism.

In Part 1 , it was revealed that over $60 \%$ of those who struggle with weight loss and have weight- related health issues cannot succeed in losing weight and getting healthy on a traditional low calorie diet. This 4 part series will finally give you the information you need to lose weight once and for all and obtain and maintain your best health.

\section{You can gain weight on a low calorie diet}

The truth is, over $60 \%$ of adults are born with the genes for developing metabolic syndrome (for ease; Metabolism B). For millions of people; skipping breakfast, fasting, and having gaps of more than 5 hours without eating can actually cause weight gain. People with Met B cannot succeed losing weight by decreasing calories and increasing activity. Therefore well -known weight loss programs like Weight Watchers ${ }^{\mathrm{mw}}$, Jenny Craig ${ }^{\mathrm{m}}{ }^{\mathrm{m}}$, Nutrisystem ${ }^{\mathrm{Tm}}$, Medifast ${ }^{\mathrm{Tm}}$ and even RD individualized low-calorie programs fail to work in the long term as any weight lost is quickly regained in the majority of dieters. It's interesting to watch the progress of Jenny Craig and Weight Watchers paid celebrities who lose weight and regain all of it after a short period of time. If these programs really work, why does everyone regain everything they lost in short order? They regain everything because they have Metabolism B and calorie based diets no longer work for them.

"Calories in-calories out" only works for those with metabolism $\mathbf{A}^{\mathrm{rm}}$

The "calories in-calories out" concept applies to less than $40 \%$ of people. When Met $\mathrm{A}^{\text {tw }}$ folks decrease calories and increase activity, they will lose weight. If they decrease their fat intake, they can reduce their cholesterol. No matter how much carbohydrate they consume, they will not develop type 2 diabetes. Something as simple as eliminating regular soda and night snacking is all that is needed to regulate a Met A's weight.

\section{Metabolism $A^{\mathrm{Tm}}$ is NOT the norm}

Be assured: Metabolism A is not the norm. Most people with weight issues have the alternate metabolism (Metabolism B). Met B is progressive and rooted in increasing levels of the fat gain hormone; insulin. Those with Metabolism A do not have a problem with insulin imbalance. When they eat carbohydrate foods, their blood glucose rises, the pancreas releases the right amount of insulin, and carbohydrates are processed normally. If they over eat calorically, they gain weight; as the "calories in-calories out" applies to them. If they cut their calorie intake back, they will lose weight.

\section{Gaining weight on a low-calorie diet}

Those with Metabolism B are born with the genetic predisposition to over-release of insulin based on any rise in blood glucose. In short, they "over" process blood glucose resulting in a growing amount of fat on their body, in their blood (cholesterol and triglycerides), and even in their liver.

If people with Metabolism B follow a low-calorie diet (low in fat and higher in carbohydrates) they will gain fat, increase cholesterol/ triglycerides, increase blood pressure, decrease Vitamin D, and experience fatigue, listlessness, irritability, and crave more carbohydrate foods. Over time, their medication needs will increase and they will gradually develop more health conditions. High insulin levels are not normal and they are the root of many health conditions and illnesses

\section{The story of the bagel bomb}

The best way to understand the very different processing of carbohydrate foods can be seen in the very different way those with Met A and Met B process a bagel (I could have chosen lentils, fruit, pizza, brown/white pasta or rice, or any carbohydrate food)

\section{Metabolism $\mathrm{A}^{\mathrm{mm}}$ and the bagel}

1. A person with Met A eats a bagel

2. The bagel converts to blood glucose

3. Blood glucose rises

4. The pancreas releases the correct amount of insulin to open the correct number of muscle, liver, and fat cells enabling glucose from the bagel to leave the blood stream and enter these cells. First, muscle and liver cells are refilled with glucose (to be stored as glycogen), and any remainder of glucose from the bagel is ushered into fat cells for storage.

5. The blood glucose of the Metabolism A person returns to normal

6. Four to five hours later, the Met A person begins to feel hungry... all is normal the body of the person with Met A.

\section{Metabolism $B^{\text {tw }}$ and the Bagel BOMB}

1. A person with Metabolism B eats a bagel

2. The bagel converts to blood glucose

3. Blood glucose rises

4. The pancreas overreacts and releases excess insulin. After the liver and muscles are re-filled with glucose (glycogen), the excess insulin opens excess fat cells.

5. When excess blood glucose enters the excess number of fat cells, an excess number of fat cells are filled with circulating glucose. This leaves glucose in the blood LOWER than normal.

6. In short order...usually about 1-2 hours after eating the bagel, the person with Metabolism B experiences lower than normal blood sugar, strong carbohydrate cravings, irritability, fatigue, brain fog... and begins searching for more carbohydrate foods.

7. If they choose to eat something (let's say they grab an apple), blood glucose rises and they temporarily feel better. But...just like with the bagel, excess insulin opens excess fat cells causing the person to get "fatter" on the body and in the blood. Blood sugar drops again and 1-2 hours after the apple, they are once again looking for carbohydrate foods. 
8. To the casual observer (like a person with Metabolism A ), it appears that this person has no will- power, is "always feeding her face", can't control herself, is always tired, lazy, unmotivated, crabby, and getting fat around the middle.

The truth is, the person with Metabolism B is in metabolic turmoil. Gradually, over a period of time, the health issues listed in Part 1 begin to progress.

\section{So, what happens when a person with Met B follows a low calorie diet?}

Megan (who unknowingly has Met B) is prescribed a 1400 calorie, low fat diet, or $\mathrm{WW}^{\mathrm{Tw}}$, or Jenny ${ }^{\mathrm{Tm}}$. She dutifully chooses egg whites, a whole grain Lite English Muffin (dry), and a cup of assorted fresh fruit (fresh melon, blueberries, and other healthy, high antioxidant containing fruit). This is a cholesterol free, fat free, low calorie breakfast.

\section{But Megan unknowingly has Metabolism B!}

The carbohydrates (whole grain Lite English muffin and fruit) will turn to blood glucose

The pancreas will over release the fat gain hormone insulin

Excess insulin will over-open fat cells

Excess blood glucose will enter fat cells

Circulating blood sugar drops lower than normal

About 1-2 hours later, Megan is famished as he/she is dealing with a lower than normal blood sugar level.

If she chooses not to eat (after-all, she's counting calories)...her liver will step up to the plate and release glycogen stores.

Blood sugar will rise from the liver release

Excess fat cells are opened and fed

Megan gets fatter

Her low blood sugar causes more hunger

She ignores her hunger (counting calories)

The liver releases again.....and.... you get the picture.

As soon as she eats her cup of lentil soup for lunch (fat free and on her diet).....the carb changes to blood sugar and her pancreas once again over releases insulin..

Megan is damned if she does and damned if she doesn't eat.

If she doesn't eat, her liver will self- feed by releasing glucose.

If she eats carb, her blood glucose will rise from the food.

When these patients tell you they are following their "diet" and you don't believe them because their weight isn't moving...think again.

\section{The low-calorie diet is failing the dieter}

Part 3 of Diane Kress' 4-part series that will answer the question” Do I have Met B?

As promised, Part 3 on my 4-part series that will change your weight, health, energy, and mental/physical "youthfulness." This segment provides solid information to help you to determine if YOU are in the $60 \%$ of US adults who cannot succeed losing weight and keeping it off or reaching and maintaining great health on a traditional diet. Even old "tried and true" diets no longer work. At the end of this series you will realize that it was not you who failed, it was the diet that failed you.

\section{Do YOU have Metabolism $B^{\text {mw }}$ ?}

Are you having difficulty losing weight? Despite solid dieting attempts, you can no longer lose weight and keep it off; even your old "tried and true" methods no longer work. Do you notice fat accumulating around your middle; belly fat, muffin top, love handles, back fat? Are you LOW on energy? Do you feel tired upon arising, need a nap or caffeine during the day, fall asleep watching TV soon after dinner? Is your overall body fat percentage increasing? Is your blood pressure inching up? Have you been told your cholesterol is high? Has your doctor mentioned your Vitamin D level is low...take a supplement? Do you notice problems with short term memory, focus, and concentration? Are you experiencing food cravings and do you find yourself binge eating? Do your moods rollercoaster between depression/anxiety? Do you have a short fuse? Do you feel low selfesteem or ashamed due to your weight or size? Dieting is a different world for those with Met A vs those with Met B.

\section{Do I have Met B?}

There are 3 ways to conclude if you have Metabolism B. Fasting lab work, personal symptoms, and medical history

\section{Fasting lab work that confirms met $B$}

Make sure to have labs drawn as close as possible to wake up after fasting a minimum of 8 hours. The following are the qualifiers for Met B. They will not match the qualifiers on your lab tests.

\begin{tabular}{|l|l|}
\hline Fasting: Met A Fasting & Labs indicating Met B \\
\hline Glucose 65-85 & Glucose: Under 65 OR over 85 \\
Hemoglobin A1C 5.3- 5.6 & Hemoglobin A1C: Under 5.3 OR over 5.6 \\
Insulin level 7.0 or under & Fasting insulin level: Over 7.0 \\
Vitamin D Over 40 & Vitamin D: Under 40 \\
Total cholesterol 200 & $\begin{array}{l}\text { Total Cholesterol: Over 200 without } \\
\text { medication }\end{array}$ \\
LDL cholesterol Under 100 & $\begin{array}{l}\text { LDL cholesterol: Over } 100 \text { without } \\
\text { medication }\end{array}$ \\
Triglycerides Under 100 & Triglycerides: Over 100 \\
TSH .45 -4.0 & TSH: Under .45 or over 4.0 \\
\hline
\end{tabular}

The symptoms of Met B

Frequent fatigue

Late afternoon energy slump

Mild depression

Mild anxiety

Occasional Panic Attacks

Cravings for carbs (chocolate, chips, bread, pasta, ice cream, etc)

Apathy

Not feeling full for any length of time

Old tried and true diets no longer work

Midsection fat deposits (muffin top, belly fat, back fat, love handles)

Difficulty losing weight and then keeping it off

Poor short term memory

Problems with focus and concentration 
Racing thoughts or brain fog

Trouble falling asleep or staying asleep

Decreased libido

Caffeine has less of an impact than it used to

Alcohol has more of an impact than it used to

Intermittent blurry vision

Difficulty with night driving and increased light sensitivity

\section{Family or Personal Medical history of those with Met B}

High blood lipids like cholesterol, LDL, triglycerides

Alzheimer's disease

Anxiety disorder or panic attacks

Cancer (breast, colon, skin, prostate, uterine, testicular, pancreatic)

Diabetes (hypoglycemia, pre diabetes, gestational diabetes, type 2 diabetes)

GERD

Sleep apnea

Heart attack

Stroke

Hypertension

Hypothyroidism

PCOS

Erectile dysfunction

Autoimmune diseases (rheumatoid arthritis)

Pancreatitis

Will you have all the lab work, symptoms, or medical history of a person with Met $B$ ?

No. Remember that Met B is a progressive condition. You might start off with normal labs and no real symptoms but as your life progresses, environmental stressors occur and your genetic predisposition causes these changes to occur.

\section{THE conclusion of Diane Kress' 4 part series. “The conclusion is your solution"}

$60 \%$ of American adults who struggle with weight and weight related issues have been fed incorrect and potentially harmful dietary advice regarding weight loss, lowering cholesterol, blood pressure, blood glucose, obesity, controlling metabolic syndrome, controlling and preventing type 2 diabetes, decreasing the risk of PCOS, preventing certain cancers, and postponing or preventing Alzheimer's disease. The first 3 parts of this 4-part series on the $60 \%$ explained the dramatic differences in the ability to lose weight and get healthy between people with Metabolism A and those with Metabolism B. These terms (Met A and Met B) are used to differentiate between people who are born with the genetic predisposition to insulin imbalance/insulin resistance and those who will not develop this hormonal imbalance.

\section{The $40 \%$ with Metabolism A}

$40 \%$ of US adults will normally process blood glucose generated from eating carbs AND the liver's natural release of glycogen. With Met A, the pancreas releases the correct amount of insulin to match a rise in blood glucose. These people, those with normal metabolism of carbs and liver glycogen, have Metabolism A. If a person with Met A is overweight and/or has high cholesterol, LDL cholesterol and blood pressure; it is most likely due to overeating calories and saturated fat and without regular exercise. If they make an effort to lose weight and lower their cholesterol and blood pressure, $40 \%$ of the population CAN succeed with any low calorie, low fat diet program.

\section{The $60 \%$ with Metabolism B}

$60 \%$ of the adult population does not have normal carbohydrate metabolism. Themajorityofpeoplewhoeventuallybecomeoverweightand overfatwereborn with thegeneticpredisposition todevelopMetabolismB. Due to an accumulation of life stressors such as high carb intake, inadequate physical activity, emotional or physical stress, pain, illness, or even certain medications; they eventually begin to over release insulin. Insulin is a fat gain hormone. Due to the combination of Met B genes plus life stressors, they begin to "over" process blood glucose into excess fat on their body and in their blood (cholesterol and triglycerides and fatty liver).

\section{Becoming a Detective}

As a registered dietitian and certified diabetes educator, I specialize in medical nutrition therapy for weight reduction, metabolic syndrome, and obesity, PCOS, pre- diabetes and type 2 diabetes.

For the first half of my 35 -year career, I was employed as an RD and CDE in hospitals, medical centers, and diabetes centers, I taught my patients the traditional diets approved by the medical associations.

I have always approached my career in a unique way. I didn't want to simply teach a prescribed diet and move on to the next patient without knowing the long-term results of the program and my patients' effort.

Over those early years, I spent extra time collecting and reviewing my patients' ongoing lab work, comparing changes in weight and body measurements, and noting any additions in types and/or doses of medications prescribed for weight related medical conditions. It became obvious that the outcomes of the low calorie, low fat diet with exercise were not making a statistical difference with weight loss, blood pressure, cholesterol, triglycerides, Vitamin D, or blood glucose.

In other words, the traditional diet for weight reduction, blood pressure, cholesterol, and blood glucose was not working... Instead of losing weight and getting healthier, more than half of our patients lost only $8-10$ pounds and then regained this weight, their medications were not decreased or eliminated, and they had only minor changes in body measurements (often increasing inches around the middle).

I promise that many RD's I worked with over 35 years did not believe their patients were following their program. But I was certain that I followed the low calorie, low fat diet...

When I began to gain weight around the middle, developed an elevation in cholesterol, blood pressure, and even blood glucose while following the perfect rendition of the low calorie, low fat diet with exercise...I was SURE there was a problem with the diet

I wasn't going to fool myself! I could no longer teach a diet protocol that I knew didn't work for over half the patients who worked with me. I decided to leave the hospital setting and open my own private practice as I was determined to dismantle the traditional diet and rebuild a program that matched most people with Metabolism B. 
I asked internists, cardiologists, endocrinologists, and gastroenterologists to consider sending their patients to me; that I was working on a very different type of diet constructed with Metabolism B in mind. They answered by giving their patients a choice... hospital diet instruction or this "new" diet plan built to match insulin imbalance.

Over a period of time, I refined the new program until it became very effective..... diet that truly matched the Met B metabolism was finally complete. Patients' weight, weight related lab work, and amount and types of medications all showed great improvement.

After years of research, development, refinement....there was finally a solution!

\section{The Solution to a Met $\mathrm{B}^{\mathrm{Tm}}$ Metabolism: The metabolism miracle by Diane Kress}

The new diet program must work to rest the over-reacting pancreas and liver. If I could get the pancreas to stop over-releasing the fat gain hormone, insulin. The pancreas and liver would take a break. During that break, a dieter would burn excess fat stores instead of bulking them up. There are 3 macro (large) nutrients: carbohydrate, protein, and fat. The only nutrient that turns $100 \%$ into blood glucose and triggers an over-release of insulin is carbohydrate. I knew that I would have to decrease carbohydrate intake as the typical American diet is about $65-70 \%$ carbohydrate. Even the traditional diet for diabetes was $55-60 \%$ carbohydrate!!!

All available low carbohydrate diets are unnecessarily VERY low in carbohydrate content. Keeping your total carb grams to under 25 grams/day makes it impossible to have good nutrition. A very low carbohydrate regimen also makes the program impossible to follow in the long-term.

I found that the pancreas does not over- release insulin after eating most vegetables. Most veggies are high in water content and fiber. (Corn, peas, beets, potatoes, and legumes are more like starch than veggies and do impact blood glucose) So, I realized that on the new program, most vegetables could be liberally consumed without counting their carbohydrate grams.

Only about $50 \%$ of protein converts into blood glucose...but the conversion time is slow and steady. Protein does not really kick the pancreas for quick insulin release. Good news! Lean, heart healthy protein could also be liberal and neutral on the new program. Only about $10 \%$ of fat converts to blood glucose. So, healthy fats like olives, olive oil, other vegetable oils, avocado, nuts, and seeds would also be neutral,

\section{Step 1 of the lifestyle program for Metabolism B:</strong}

\section{Step 1}

\section{Duration: Eight weeks or more}

The Challenge; A lower carbohydrate period (not a typical low carb diet) designed to rest the overworking pancreas and liver while decreasing fat in the blood (cholesterol and triglycerides, liver fat stores) as well as fat on the body (belly fat, muffin top, back fat)

The Results; Lose a specific amount of fat and inches at the end of each 8 -week period of time. Within 8 weeks, you will experience marked improvement in weight, body fat, blood pressure, cholesterol, triglycerides, insulin, insulin resistance, Vitamin D, and blood glucose. Energy level soars, focus and concentration improves, and your mood is stable.
Cravings for carbohydrates are gone, over-eating is a thing of the past, and for the first time in a long time, you feel in control of your weight, health, and life. I also found that a maximum of 5 grams net carb from blatant carb (like real popcorn, Greek yogurt, whole grain crackers, fruit, etc) can be placed at every meal and bedtime without causing significant insulin release. And, if you are willing to exercise for 40 or minutes at a time (a walk, bike ride, swim, yoga, Tai Chi, run), you can also have a piece of fresh fruit right before the workout!

\section{Step 2}

Time: After spending at least eight weeks in Step 1, feel free to move to 8 weeks or more in Step 2. You can remain in Step 2 until you reach your desired weight.

\section{The Duration: 8 weeks or longer, if desired}

The Challenge: Re-introduce carbohydrate foods of the right type, in the right amount, at the right time to promote continued fat burning and weight loss while gently restarting the pancreas and liver.

The Results: Steady and predictable fat and inch loss, continued improvement in lab work and decreases in weight- related medications, high energy, no cravings, and normal appetite.

The next obstacle was how to transition a person from being on a reduced carbohydrate Step (Step 1) to eating a controlled amount of carbohydrate (Step 2) without over-stimulating the rested pancreas and liver and causing weight to regain. All other low carb diets fail as soon as the dieter attempts to add carbohydrate back into their intake. Step 2 is the hallmark of diet for Met Step 2 is the "Missing Link" not built into low carb programs. If carbohydrate is not reintroduced correctly, the pancreas and liver will once again over-react with excess insulin and glycogen release. It turns out that Step 2 needed 3 separate components: Carbohydrate foods had to be of the right type, in the right amount, at the right time.

Type of carbohydrate: The first carbohydrate reintroduced needed to be low glycemic index. I chose carbohydrates that had a maximum of about 50 on the GI scale with lots of fiber. So, it's not that carbohydrate foods can be reintroduced into the diet haphazardly after Step 1; you have to insert the right type of carbs. There must be a gentle reintroduction of carbohydrate foods.

Amount of carbohydrate: This part of the program took the longest time to calculate. When the dust settled, I found that 11-20 grams net carb was the appropriate amount of carbohydrate for breakfast, lunch, dinner, bedtime, middle of the night (if awake) and between any meals that exceed 5 hours. The fiber needed to be a minimum of 2 grams per serving. The sugar needed to be 6 grams or less per serving.

The timing of eating carbohydrates is also important. Have the right amount of carb within one hour of wake up, at all meals, bedtime, and between meals that are five hours or more apart.

I made a list of all the foods that fit this bill. So, pick one of the carbs on the list in the right amount and place them accordingly:

Sample Day on Step 2

Wake up: 7:00AM

Breakfast: 8:00AM with 11-20 grams net carb and neutrals

Lunch: 12:30-1:00PM with 11-20 grams net carb and neutrals

Snack: neutral foods like cheese stick or almonds 
Dinner: 5:30PM with 11-20 grams net carb and neutrals

Night snack: 10:00PM with 11-20 grams net carb and neutrals

Bedtime: 10:45PM Sleep through the night or, if you are awake: 1120 grams net carb snack

If you are hungry at any time, neutral food choices are fine!

\section{Step 3}

Time: This is your "lifetime" Step for weight and health maintenance. Metabolism B is never "cured" as it is part of your genetic makeup. You are eating and exercising in a way that causes your body to react normally to blood glucose and glycogen release.

The Duration: Lifetime Lifestyle

The Challenge: Increasing choices of carbohydrate in an amount that maintains your desired weight while maintaining normal lab values, weight, and body measurements. Continue a lifetime of energy, focus/concentration, stable moods, normal appetite, and control of weight and health

Results: A lifetime of desired weight, normal lab values on as little medication as possible. When a person has reached his desired weight, everyone has a different amount of recommended carbohydrate grams/ day, spread out to avoid gaps of over 5 hours without eating. The amount of carb grams per day is based on gender, height, desired weight, and activity. If you take a vacation, celebrate the holidays, attend a special event that causes you to go "off program" you can always return to Step 1 for two weeks followed by Step 2 for two weeks and then return to Step 3!

\section{The kook}

The Metabolism Miracle was first released in hardcover 2009. In less than 3 months it became a NY Times Bestseller and topped the health and wellness charts in all markets.
An updated version of The Metabolism Miracle, Revised Edition was released in 2016.

In 2011, The Metabolism Miracle for direct mail was published by Rodale Books. The book is available around the world and is licensed in 8 languages. The Metabolism Miracle Cookbook was released in 2010. A unique feature of the MM Cookbook is that its first 100 pages are a condensed and simplified summary of the Metabolism Miracle. The 175 recipes in The Metabolism Miracle Cookbook matches The Metabolism Miracle and The Diabetes Miracle

In 2011, The Diabetes Miracle was released in hardcover. In 2012, The Diabetes Miraclepaperbackwasreleased. TheDiabetes Miraclecontainsthe entire dietand lifestyleprogram that is the core of The Metabolism Miracle. When "The Metabolism Series" was published over a 3-year period, hundreds of thousands of people began "living the Miracle".

In May 2016, the revised edition of "The Metabolism Miracle” was released in paperback! The core program is the same, but it contains all the little tweaks and improvements that occurred since the original release!

In April, 2010, a subscription support website called Miracle-Ville. com [1] was launched to provide tips, recipes, tweaks, and support. The Miracle series author, Diane Kress is regularly in Miracle-Ville.com to personally answer questions and concerns. Through the years, I've always kept my finger on the pulse of my readers paying close attention to new food offerings, questions, comments, and queries regarding the programs. All information on Miracle-Ville.com [1] is checked for accuracy and updated as needed.

\section{References}

1. http://www.Miracle-Ville.com
2. Metabolism Miracle Book website http://www.themetabolismmiracle.com
3. Diane Kress' blog http://www.dianekress.wordpress.com

Copyright: (C2017 Kress D. This is an open-access article distributed under the terms of the Creative Commons Attribution License, which permits unrestricted use, distribution, and reproduction in any medium, provided the original author and source are credited. 